\title{
The Relationships between Addiction to Highly Caffeinated Drinks, Burnout, and Attention-Deficit/ Hyperactivity Disorder
}

\author{
Eun Ju Bae', Eun Bi Kim², Bo Ram Choi', Sun Ho Won', Ji Hwan Kim², \\ Sun Min Kim², Hyun Jeong Yoo', Sung Man Bae', and Myung Ho Lim, \\ ${ }^{1}$ Department of Psychology, Graduate School, Dankook University, Cheonan, Korea \\ ${ }^{2}$ Department of Psychology, College of Social Science, Dankook University, Cheonan, Korea
}

\begin{abstract}
Objectives: This study aimed to evaluate the addiction to highly caffeinated drinks among university students, and we investigated the relationships between smartphone addiction, depression, anxiety, attention-deficit/hyperactivity disorder (ADHD), burnout, self-esteem, impulsiveness in high caffeine drink addiction risk group (high caffeine group). We also compared these mental health factors differences between the high caffeine group and the control group; and, investigated the relative risk between the independent variables of the high caffeine group.

Methods: This study was conducted in Korea, from June 2015 to July 2016. A set of questionnaires was administered on 511 college students.

Results: The participants who belonged to the high caffeine group were more likely to demonstrate the symptoms of ADHD and higher levels of burnout and impulsiveness. Further, the results of logistic regression analysis confirmed the association between the high caffeine group and burnout.

Conclusion: These behaviors among university students addicted to highly caffeinated drinks suggests the need for timely and effective interventions for those at risk of addiction.
\end{abstract}

Key Words: Caffeine addiction; Depression; Attention-deficit/hyperactivity disorder; Burnout; Impulsiveness.

Received: May 15, 2019 / Revision: June 17, 2019 / Accepted: June 26, 2019

Address for correspondence: Myung Ho Lim, Department of Psychology, College of Social Science, Dankook University, 119 Dandae-ro, Dongnam-gu, Cheonan 31116, Korea

Tel: +82-41-550-3263, Fax: +82-41-559-7852, E-mail: paperose@dku.edu

\section{INTRODUCTION}

In recent times, highly caffeinated drinks, which are marketed as beverages that can alleviate fatigue and provide stimulation, have become popular among and are abused by teenagers and young adults [1]. In addition, some young adults consume a mixture of highly caffeinated drinks and other sports drinks to hasten the effects of highly caffeinated drinks. They may also mix highly caffeinated drinks and alcohol to create cocktails. In a survey that was conducted among 300 college students in $2014,68.7 \%$ of the students reported that they had consumed highly caffeinated energy drinks [2]. A highly caffeinated drink is a beverage that contains more than $0.15 \mathrm{mg}$ of caffeine per $\mathrm{mL}$ [3]. Quintessential highly caffeinated drinks such as Burn Intense $(250 \mathrm{~mL})$, Red Bull $(250 \mathrm{~mL})$,

This is an Open Access article distributed under the terms of the Creative Commons Attribution Non-Commercial License (https://creativecommons.org/licenses/by-nc/4.0) which permits unrestricted non-commercial use, distribution, and reproduction in any medium, provided the original work is properly cited. and Hot Six $(250 \mathrm{~mL})$ contain $30 \mathrm{mg}, 63 \mathrm{mg}$, and $60 \mathrm{mg}$ of caffeine, respectively [1].

In general, the intake of small amounts of caffeine can temporarily alleviate mild fatigue and boredom, and provide stimulation with almost no harmful effects. However, the excessive or continuous consumption of caffeine can cause psychological and physical side effects. In a study that was conducted in Seoul, college students reported that the most frequent side effect of consuming energy drinks is an abnormal heart rate/rhythm. Further, they reported that addiction was the most significant problem that was associated with the consumption of energy drinks [4]. Gunja and Brown [5] reported that an accelerated heart rate, agitation, and gastrointestinal disturbances are common side effects of consuming highly caffeinated drinks and that caffeine addiction is becoming more prevalent, especially among young adults.

Kendler et al. [6] reported that an addiction to highly caffeinated energy drinks is significantly correlated with depres- 
sion. Park et al. [7] reported that students who did not consume highly caffeinated energy drinks had a $24.7 \%$ probability of experiencing depression. Further, $54.9 \%$ of the students who consumed these drinks more than 5 times a week experienced depression. This suggests that highly frequent consumption of highly caffeinated drinks is associated with a higher likelihood of experiencing depression. In one study that was conducted among college students, the consumption of energy drinks was significantly correlated with the symptoms of anxiety [8].

In a study that was conducted among elementary school students in Korea, those with attention-deficit/hyperactivity disorder (ADHD) consumed higher levels of caffeine than normal controls [9]. Similarly, Martin et al. [10] found that high levels of caffeine intake are correlated with the symptoms of ADHD and conduct disorder, and smoking and aggressive behaviors. Kendler et al. [6] found that excessive caffeine intake is associated with greater risk of developing an externalizing disorder [odds ratio (OR) 1.94-2.34] rather than an internalizing disorder (OR 1.50-1.79). Heinz et al. [11] found that the habitual intake of caffeinated alcoholic beverages is positively correlated with impulsivity and risk behaviors. Evren and Evren [12] also reported positive correlations between the consumption of energy drinks, self-injurious behaviors, and suicidal impulses. On the other hand, caffeine addiction is a substance addiction, and multiple addictions can simultaneously coexist as comorbidities. Indeed, it has been reported that addiction to substances such as caffeine and cigarettes are correlated with behavioral addictions (e.g., addiction to smartphones, internet, gambling, and eating). The number of studies of smartphone addiction is continuously increasing; however, only a few studies have examined the phenomenon of coexisting substance and behavioral addictions. In a study that was conducted among medical students, the consumption of energy drinks was significantly correlated with burnout [13]. Another experimental study found that the consumption of energy drinks can greatly increase burnout during marathons by increasing concentration, energy, and decreasing fatigue [14]. In another study, intake of highly caffeinated drinks was related to self-esteem [15]. Addiction to highly caffeinated drinks is also a risk factor for obesity, and addiction to energy drinks is positively correlated with marijuana use, sexual risk behaviors, fighting, failure to wear a seatbelt, reckless risk behaviors, smoking, drinking, and illegal drug use among Caucasians [16,17].

The present study had four objectives: 1) to examine addiction to highly caffeinated drinks among college students; 2) to investigate the relationship that addiction to highly caffeinated drinks shares with smartphone addiction, depression, anxiety, symptoms of ADHD, burnout, self-esteem, and impulsiveness; 3) to compare the differences in mental health characteristics between individuals who are at high risk for addiction to highly caffeinated drinks and individuals in the control group; and 4) to investigate the relative risk of the various variables in the high caffeine drink addiction risk group.

\section{METHODS}

\section{Subjects}

This study was conducted from June 2015 to July 2016 among 511 college students who agreed to participate in the survey after they were briefed about the purpose of the study. Basic demographic information (e.g., age, sex, academic achievements) were collected from the participants. This random sample of participants was recruited from various departments of a university that is located in Cheonan. Participants who consumed one or more highly caffeinated drinks per week were assigned to the high caffeine drink addiction risk group or the high caffeine group (i.e., individuals who were at risk for addiction to highly caffeinated drinks) and the others were assigned to the control group. In a previous research study that used data that were collected by the Ministry of Gender Equality and Family to examine the frequency of consumption of highly caffeinated drinks among Korean high school students, $14.7 \%$ of the students reported that they had consumed highly caffeinated drinks once or twice a week. This is similar to the percentage (17.6\%) of participants who were assigned to the high caffeine group [18]. Informed consent was obtained from all the participants, and the research method of the present study was reviewed and approved by Dankook University Ethics Committee (DK2017-02-004).

\section{Procedure}

After the participants provided written informed consent, they responded to an ethically approved and individually administered questionnaire. We requested the participants complete the surveys within or near a university campus or around bus stops.

\section{Measures}

\section{The epidemiology and consumption of highly caffeinated} drinks

Each student responded to a questionnaire that consisted of items that required information about their sex, age, grade, recent academic achievements, types of highly caffeinated drinks that they consumed, and their frequency of consumption. 


\section{Korean Adult Attention-Deficit/Hyperactivity Disorder Scale}

The Adult Attention-Deficit/Hyperactivity Disorder Scale (AADHDS) is a self-report assessment that has been developed by Murphy and Barkley [19] based on the Diagnostic and Statistical Manual of Mental Disorders-IV (DSM-IV) [20] diagnostic criteria for ADHD in adults. This 18-item scale is a valid measure that can differentiate between adults with and without ADHD. It is designed to effectively differentiate between three subtypes of ADHD: predominantly inattentive, predominantly hyperactive-impulsive, and combined (i.e., hyperactive-impulsive and inattentive). The Korean standardization of this assessment (K-AADHDS) was undertaken by Kim [21]. The internal consistency (Cronbach's a) of this scale was 0.80 in the present study. The internal consistency coefficients (Cronbach's $\alpha$ ) were $0.84,0.80$, and 0.80 for the inattentiveness, hyperactivity, and impulsiveness subscales, respectively.

\section{Korean Smartphone Addiction Scale}

The Korean Smartphone Addiction Scale (KSAS) was developed in 2011 by the National Information Society Agency to measure smartphone addiction and use. It was developed based on the Internet Addiction Scale and Cellular Phone Addiction Scale [22]. The KSAS, which has been designed for use with adults, consists of a total of 15 questions. Five questions assess difficulties with daily activities, two questions assess the pursuit of a virtual world, four questions assess withdrawal, and four questions assess tolerance. Responses are recorded on a four-point scale, and the composite score can range from 15 to 60 points. In a study of 3230 adults, by the National Information Society Agency, the high caffeine group accounted for $1.0 \%$, and the potential risk group for $6.7 \%$, for a total of $7.7 \%$ in the addiction group. The internal consistency (Cronbach's $\alpha$ ) of this scale was 0.81 in the original validation study and 0.89 in this study.

\section{Beck Depression Inventory}

This Beck Depression Inventory (BDI) was developed to measure depression in adults, and it is widely used around the world. It consists of 21 items that assess the cognitive, emotional, and physical symptoms of depression. Respondents are required to choose one of several sentences that differentially describe the severity of a symptom. Such a response method is more advantageous than Likert scales, which can confuse respondents by requiring them to quantify their psychological states. Scores that are assigned to the responses can range from 0 to 3 for each item. Higher scores are indicative of a greater severity of depression. Lee and Song [23] translated the BDI in Korea. The CRESCEND study es- tablished that scores of 10-15 were indicative of a mild depressive state, 16-23 of a moderate depressive state, and 24-63 of a severe depressive state. The internal consistency (Cronbach's $\alpha$ ) of this scale was 0.89 in their study [23] and 0.89 in the present study.

\section{Beck Anxiety Inventory}

The Beck Anxiety Inventory (BAI) is a useful measure of clinical anxiety. It can be used to measure anxiety among adults who have never been diagnosed with a psychiatric disorder. It has been translated into Korean by Yook and Kim [24]. The internal consistency of the Korean version of the BAI was found to be 0.91 . Scores that lie between 22 and 26, and 27 and 31 are indicative of mildly and considerably high levels of anxiety; scores that are equal to or higher than 32 are indicative of extremely high levels of anxiety. The internal consistency (Cronbach's $\alpha$ ) of this scale was 0.82 in this study.

\section{Maslach Burnout Inventory}

The Maslach Burnout Inventory (MBI) consists of items that assess emotional exhaustion, depersonalization, and a perceived lack of personal accomplishment. Kang and Kim [25] reported an internal consistency coefficient (Cronbach's a) of 0.85 for a Korean sample. Each of the 22 items are to be rated on a scale that ranges from 0 to 6 . Higher scores are indicative of higher levels of burnout. According to the Official Manual of Manual Worker, scores that are equal to or higher than 27 points are indicative of emotional exhaustion. The internal consistency (Cronbach's $\alpha$ ) of this scale was 0.84 in the present study.

\section{Rosenberg's Self-Esteem Scale}

The Rosenberg's Self-Esteem Scale (RSE) measures selfesteem. The inventory was adapted by Lee [26] for use with Korean samples. This self-report assessment consists of a total of 10 questions (five questions are positively worded, and five questions are negatively worded). Each item can receive a score that ranges from 0 to 3 points, and the total score can range from 0 to 30 points. The internal consistency (Cronbach's a) of this scale was 0.88 in this study.

\section{Barratt Impulsiveness Scale}

The Barratt Impulsiveness Scale (BIS) was developed to measure of impulsiveness. Lee [27] adapted it to the Korean context. It consists of 23 questions and three subordinate factors: six questions measure cognitive impulsiveness, eight questions measure motor impulsiveness, and nine questions measure non-planning impulsiveness. Responses are recorded on a four-point Likert-type scale that ranges from "It never does" (1 point) to "It always does" (4 points). Higher scores are in- 
dicative of higher levels of impulsiveness. The internal consistency (Cronbach's $\alpha$ ) of this scale was 0.82 in the Korean validation study and 0.81 in the present study.

\section{Data analysis}

We used the Statistical Package for the Social Sciences (SPSS) 24 software (IBM Corp., Armonk, NY, USA). We conducted t-tests and chi-squared tests to examine age and sex differences in epidemiologic variable. Finally, through correlation analysis, we investigated the relationship between addiction to highly caffeinated drinks and mental health indicators. Analysis of covariance (ANCOVA) was used to examine differences in the symptoms of ADHD, smartphone addiction, depression, anxiety, burnout, impulsiveness, and self-esteem between the high caffeine and control groups. Additionally, logistic regression analysis was used to examine the relative risk that each of these variables entailed. Results with a p-value that is less than 0.05 were considered statistically significant.

Table 1. Epidemiological characteristics between high caffeine group and comparison group

\begin{tabular}{lcccc}
\hline & $\begin{array}{c}\text { High caffeine } \\
\text { group }(\mathrm{n}=92)^{*}\end{array}$ & $\begin{array}{c}\text { Control group } \\
(\mathrm{n}=419)\end{array}$ & tor $\chi^{2}$ & p value \\
\hline $\begin{array}{l}\text { Age }(\mathrm{yr}) \\
\text { Sex }\end{array}$ & $22.74 \pm 5.60$ & $22.96 \pm 7.36$ & -0.26 & 0.792 \\
$\quad$ & & & $6.56^{\dagger}$ & 0.001 \\
Women & $38(41.3)$ & $239(57.0)$ & & \\
$\quad$ Men & $52(56.5)$ & $180(43.0)$ & & \\
$\begin{array}{l}\text { Economic } \\
\text { status }{ }^{\ddagger}\end{array}$ & & & 0.36 & 0.840 \\
Low & $6(6.5)$ & $30(7.4)$ & & \\
Middle & $40(43.4)$ & $169(41.5)$ & & \\
High & $43(46.7)$ & $208(51.1)$ & & \\
\hline
\end{tabular}

Data represent mean \pm standard deviation, by independent $t-$ test, or $\mathrm{n}(\%)$, by chi-square test. *high caffeine group: high caffeine drinking addiction risk group, ${ }^{\dagger} p<0.01$, " $\mathrm{KRW} /$ month, middle: $2000000-4000000 \mathrm{KRW} /$ month, high: above $4000000 \mathrm{KRW} /$ month

\section{RESULTS}

\section{Demographic characteristics of the participants}

The sample consisted of 511 men $(\mathrm{n}=234,45.7 \%)$ and women ( $n=278,54.3 \%)$. A total of 92 (18.0\%) participants belonged to the high caffeine group (men: $n=52,56.5 \%$; women: $n=38$, $41.3 \%)$. The control group consisted of 180 (43.0\%) men and $239(57.0 \%)$ women. There was a significant difference in the sex ratios of the two groups $\left(\chi^{2}=6.56, \mathrm{p}<0.01\right)$. The mean ages of the participants who belonged to the high caffeine and control groups were $22.74 \pm 5.60$ years and $22.96 \pm 7.36$ years, respectively. There was no significant age difference between the two groups (Table 1).

\section{Correlations between the high caffeine group, smartphone addiction, depression, anxiety, ADHD, burnout, self-esteem, and impulsiveness}

Depression $(\mathrm{r}=0.108, \mathrm{p}<0.05)$ and burnout $(\mathrm{r}=0.128, \mathrm{p}<0.01)$ were positively correlated with the high caffeine group. Although the p-value is significant, the magnitude of the correlation coefficient is small, meaning although the correlation is significant between the two factors, it is not a strong relationship. In addition, depression, anxiety, ADHD, burnout, and impulsiveness were positively inter-correlated. Self-esteem was negatively correlated with variables such as smartphone addiction $(\mathrm{r}=-0.262, \mathrm{p}<0.01)$, depression $(\mathrm{r}=-0.267$, $\mathrm{p}<0.01)$, anxiety $(\mathrm{r}=-0.583, \mathrm{p}<0.01), \operatorname{ADHD}(\mathrm{r}=-0.270, \mathrm{p}<0.01)$, burnout $(\mathrm{r}=-0.529, \mathrm{p}<0.01)$, and impulsiveness $(\mathrm{r}=-0.412, \mathrm{p}<$ 0.01) (Table 2).

\section{Comparison of the high caffeine and control groups}

The high caffeine and control groups obtained total scores of $32.88 \pm 0.80$ and $32.15 \pm 0.37$ on the KSAS, respectively. The mean difference was nonsignificant. The high caffeine and control groups obtained total scores of $9.56 \pm 0.79$ and $8.13 \pm$ 0.37 on the BDI, respectively; the mean difference was non-

Table 2. Correlation coefficient of high caffeine, smartphone addiction, depression, anxiety, ADHD, exhaustion, impulsivity and selfesteem of life of high caffeine group and control group

\begin{tabular}{lccccccc}
\hline & 1 & 2 & 3 & 4 & 5 & 6 & 7 \\
\hline 1. High caffeine & & & & & & & \\
2. KSAS & -0.008 & & & & & & \\
3. BDI & $0.108^{*}$ & $0.349^{\dagger}$ & & & & & \\
4. BAI & 0.051 & $0.242^{\dagger}$ & $0.377^{\dagger}$ & & & & \\
5. K-AADHDS & 0.038 & $0.204^{\dagger}$ & $0.446^{\dagger}$ & $0.522^{\dagger}$ & & & \\
6. MBI & $0.128^{\dagger}$ & $0.271^{\dagger}$ & $0.366^{\dagger}$ & $0.515^{\dagger}$ & $0.386^{\dagger}$ & & $-0.270^{\dagger}$ \\
7. RSE & -0.038 & $-0.262^{\dagger}$ & $-0.267^{\dagger}$ & $-0.583^{\dagger}$ & $-0.529^{\dagger}$ & $-0.412^{\dagger}$ \\
8. BIS & 0.075 & $0.312^{\dagger}$ & $0.538^{\dagger}$ & $0.316^{\dagger}$ & $0.311^{\dagger}$ & $0.380^{\dagger}$ & \\
\hline
\end{tabular}

Data present correlation coefficient by a correlation analysis. ${ }^{*} p<0.05,{ }^{\dagger} p<0.01$. BAI: Beck Anxiety Inventory, BDI: Beck Depression Inventory, BIS: Barratt Impulsiveness Scale, High caffeine: high caffeine addiction risk group, K-AADHDS: Korean Adult Attention-Deficit/Hyperactivity Disorder Scale, KSAS: Korean Smartphone Addiction Scale, MBI: Maslach Burnout Inventory, RSE: Rosenberg's SelfEsteem Scale 
significant. The high-risk and control groups obtained total scores of $8.20 \pm 0.88$ and $6.96 \pm 0.41$ on the BAI, respectively. The mean difference was nonsignificant.

The high caffeine and control groups obtained total scores of $30.24 \pm 0.84$ and $27.79 \pm 0.39$ on the K-AADHDS, respectively; the mean difference was significant $(\mathrm{F}=7.03, \mathrm{p}<0.01)$. The high caffeine and control groups obtained total scores of $15.44 \pm 0.45$ and $14.48 \pm 0.21$ on the inattentiveness subscale of the K-AADHDS, respectively; the mean difference was nonsignificant. The high caffeine and control groups obtained total scores of $14.80 \pm 0.45$ and $13.31 \pm 0.21$ on the hyperactivity subscale of the K-AADHDS, respectively; the mean difference was significant $(\mathrm{F}=8.92, \mathrm{p}<0.01)$.

The high caffeine and control groups obtained total scores of $66.06 \pm 2.05$ and $59.08 \pm 0.95$ on the MBI, respectively; the mean difference was significant $(\mathrm{F}=9.49, \mathrm{p}<0.01)$. The high caffeine and control groups obtained total scores of 28.55 0.56 and $29.41 \pm 0.26$ on the RSE, respectively; the mean difference was nonsignificant. The high caffeine and control groups obtained total scores of $53.74 \pm 0.98$ and $51.30 \pm 0.45$ on the $\mathrm{BIS}$, respectively; the mean difference was significant $(\mathrm{F}=5.12$, $\mathrm{p}<0.05$ ) (Table 3).

\section{Logistic regression analysis of mental health characteristics associated with the highly caffeinated drinks}

In the logistic regression model that included the high caffeine and control group, the relative risk of burnout was 2.70 times higher (95\% confidence interval $=1.51-4.80)$, and this result was statistically significant $\left(\chi^{2}=11.335, \mathrm{p}<0.01\right)$. The risk was 1.97 times higher among men than among women $(95 \%$ confidence interval $=1.21-3.21)$, and this result was also statistically significant $\left(\chi^{2}=7.336, \mathrm{p}<0.01\right)$ (Table 4).

\section{DISCUSSION}

This study provides empirical evidence about the status of addiction to highly caffeinated drinks and associated indicators of mental health. In this study, which was conducted among college students, $48.8 \%$ of the participants reported that they had consumed a highly caffeinated drink at least once. Among them, $17.6 \%$ consumed more than one can per week, and $2.8 \%$ consumed more than one can per day. These results are similar to the statistics that were released by the Ministry of Gender Equality and Family in 2019. Specifically, they reported that $14.7 \%$ of high school students consume more than one can of a highly caffeinated drink per week and that $5.6 \%$ of high school students consume more than one can of a highly caffeinated drink per day. "There was a significant sex difference in the consumption of highly caffeinated drinks" This result is consistent with Park et al. [7] reported that men are significantly more likely to consume highly caffeinated energy drinks than women. This suggests that men prefer and consume highly caffeinated drinks to a greater extent than women. Advertisements for highly caffeinated drinks utilize terms such as "power" and "energy"; therefore, due to

Table 3. Score of smartphone addiction, depression, anxiety, ADHD, exhaustion, impulsivity, self-esteem of high caffeine group and control group

\begin{tabular}{|c|c|c|c|c|}
\hline Rating scale & High caffeine group $(n=92)$ & Control group $(n=419)$ & $\mathrm{F}$ & $p$ value \\
\hline KSAS & $32.88 \pm 0.80$ & $32.15 \pm 0.37$ & 0.68 & 0.410 \\
\hline Disability of daily living & $11.33 \pm 0.31$ & $11.34 \pm 0.14$ & 0.001 & 0.980 \\
\hline Virtual world orientation & $3.34 \pm 0.12$ & $3.08 \pm 0.05$ & $4.15^{*}$ & 0.040 \\
\hline Prohibition & $8.40 \pm 0.26$ & $8.04 \pm 0.12$ & 1.52 & 0.220 \\
\hline Tolerance & $9.82 \pm 0.26$ & $9.70 \pm 0.12$ & 0.19 & 0.660 \\
\hline $\mathrm{BDI}$ & $9.56 \pm 0.79$ & $8.13 \pm 0.37$ & 2.70 & 0.100 \\
\hline $\mathrm{BAl}$ & $8.20 \pm 0.88$ & $6.96 \pm 0.41$ & 1.64 & 0.200 \\
\hline K-AADHDS & $30.24 \pm 0.84$ & $27.79 \pm 0.39$ & $7.03^{\dagger}$ & 0.008 \\
\hline Inattention & $15.44 \pm 0.45$ & $14.48 \pm 0.21$ & 3.70 & 0.055 \\
\hline Hyperactivity & $14.80 \pm 0.45$ & $13.31 \pm 0.21$ & $8.92^{\dagger}$ & 0.003 \\
\hline$M B I$ & $66.06 \pm 2.05$ & $59.08 \pm 0.95$ & $9.49^{\dagger}$ & 0.002 \\
\hline RSE & $28.55 \pm 0.56$ & $29.41 \pm 0.26$ & 1.97 & 0.160 \\
\hline BIS & $53.74 \pm 0.98$ & $51.30 \pm 0.45$ & $5.12^{*}$ & 0.020 \\
\hline Recognition & $14.98 \pm 0.28$ & $14.33 \pm 0.13$ & $4.52^{*}$ & 0.030 \\
\hline Exercise & $18.15 \pm 0.48$ & $17.02 \pm 0.22$ & $4.52^{*}$ & 0.030 \\
\hline Unplanned & $20.61 \pm 0.44$ & $19.95 \pm 0.20$ & 1.86 & 0.170 \\
\hline
\end{tabular}

Data represent mean \pm standard deviation, ANCOVA adjusted for sex by general linear model. ${ }^{*} p<0.05,{ }^{\dagger} p<0.01$. BAI: Beck Anxiety Inventory, BDI: Beck Depression Inventory, BIS: Barratt Impulsiveness Scale, F: female, K-AADHDS: Korean Adult Attention-Deficit/Hyperactivity Disorder Scale, KSAS: Korean Smartphone Addiction Scale, RSE: Rosenberg's Self-Esteem Scale 
Table 4. Logistic regression analysis of mental health characteristics associated with the highly caffeinated drinks

\begin{tabular}{lccccc}
\hline \multicolumn{1}{c}{ Variables } & Parameter stimate & Standard error & $\chi^{2}$ & p value & $\begin{array}{c}\text { Odds ratio } \\
\text { (95\% confidence interval) }\end{array}$ \\
\hline KSAS & -0.034 & 0.288 & 0.014 & 0.905 & $0.97(0.55-1.70)$ \\
BDI & 0.088 & 0.334 & 0.070 & 0.792 & $1.09(0.57-2.10)$ \\
BAI & 0.327 & 0.308 & 1.128 & 0.288 & $1.39(0.76-2.54)$ \\
K-AADHDS & -0.093 & 0.299 & 0.097 & 0.755 & $0.91(0.51-1.64)$ \\
MBI & 0.992 & 0.295 & 11.335 & 0.001 & $2.70(1.51-4.80)^{*}$ \\
RSE & -0.356 & 0.315 & 1.277 & 0.258 & $0.70(0.38-1.30)$ \\
BIS & 0.080 & 0.295 & 0.074 & 0.785 & $1.08(0.61-1.93)$ \\
Sex & 0.676 & 0.250 & 7.336 & 0.007 & $1.97(1.21-3.21)^{*}$ \\
\hline
\end{tabular}

*p<0.01. BAI: Beck Anxiety Inventory, BDI: Beck Depression Inventory, BIS: Barratt Impulsiveness Scale, K-AADHDS: Korean Adult Attention-Deficit/Hyperactivity Disorder Scale, KSAS: Korean Smartphone Addiction Scale, RSE: Rosenberg's Self-Esteem Scale

their implicit association with physical activity, these products may be more appealing to men than to women.

In this section, the important results of the study are summarized, and possible explanations for the findings are discussed. First, burnout is moderately related to addiction to highly caffeinated drinks. Accordingly, in the present study, there was a significant difference in the scores that the high caffeine and control groups obtained on the measure of burnout. Thus, these results indicate that the high caffeine group is associated with burnout. The factors that motivate college students to consume highly caffeinated drinks are primarily academic in nature [28]. Therefore, it can be suggested that academic factors are moderately related to the consumption of highly caffeinated drinks among college students. In particular, the results of the logistic regression analysis showed that there was a positive relationship between burnout and high caffeine group. This indicates that people with high levels of stress exhibit the symptoms of burnout. Consequently, they may frequently consume highly caffeinated drinks to alleviate their fatigue and sleepiness.

Second, addiction to highly caffeinated drinks is associated with externalizing disorders such as ADHD and variable such as impulsiveness. In this study, the high caffeine and control groups differed significantly in their symptoms of ADHD. This suggests that individuals with greater symptoms of ADHD are more likely to consume highly caffeinated drinks. This result is similar to past findings that the amount of caffeine that was consumed by upper elementary students with ADHD was 1.6 times higher than what was consumed by their normal counterparts. Similarly, teenagers with ADHD also consume twice as many caffeinated drinks as their normal counterparts $[9,29]$. In addition, the results of the present study are also consistent with past findings that excessive caffeine intake increases the risk of developing externalizing rather than internalizing disorders. This indicates that the effect of caffeine on the body may be more easily exhibited by externalizing rather than internalizing symptoms [6]. The con- sumption of highly caffeinated drinks may temporarily enhance concentration, but prolonged consumption can lead to negative consequences such as memory impairment. In other words, the results of this study suggest that addiction to highly caffeinated drinks is related to ADHD and that it can consequently affect the concentration circuits in the brain.

In this study, the high caffeine and control groups differed significantly on impulsiveness. This suggests that higher levels of impulsiveness are associated with a greater likelihood of consuming highly caffeinated drinks. Since impulsiveness is characterized by a preference for immediate and stimulating rewards, it may be linked to a tendency to impulsively consume reactive stimulants such as highly caffeinated drinks.

Third, the present findings suggest that an addiction to highly caffeinated drinks is unrelated to emotional factors such as depression, anxiety, and low self-esteem. The high caffeine and control groups did not differ in their levels of depression. Although burnout was strongly related to depression in previous studies, group differences in this study were not significant [30]. When compared to depression, chronic fatigue or burnout has stronger implications for social functioning and weaker implications for emotional functioning [31]. Therefore, people who consume highly caffeinated drinks may be more likely to exhibit difficulties in social functioning (e.g., physical functions and vitality) rather than emotional functioning. The high caffeine and control groups did not differ in their levels of anxiety; this result contradicts past findings [32].

The present study has a few limitations. First, the study variables were measured using self-report questionnaires; therefore, the measurements may have entailed a false negative or positive bias. Although it was important to control the confounding influence of demographic factors (e.g., socioeconomic status, period of education) on the findings, the data that were collected were insufficient to conduct these analyses. Second, as this was a cross-sectional study, this study was not able to present a causal relationship between the comparison 
group and the control group; hence, we only presented the relationship between the high caffeine drink addiction risk group, ADHD, burnout, and impulsiveness. Third, this study focused only on students who were living within the local community. Specifically, this study was conducted in a new town that has a population of more than 500000 people. Thus, the sample may not have been representative of diverse local communities. Fourth, high caffeine group was predicated upon consumption of more than one can of a highly caffeinated drink per week. However, a wide range of criteria have been used in different studies. We used the aforementioned criteria based on the data that have been released by the Ministry of Gender Equality and Family of Korea. The size of the highrisk group was small. Future studies should use larger samples to overcome this limitation. Despite these limitations, this study meaningfully examined the relationship between the consumption of highly caffeinated drinks and indicators of mental health among Korean college students. In the present study, the mental health of individuals who consume highly caffeinated drinks was assessed using universally validated measures of related constructs. Future studies should aim to address the many limitations of the present study and examine addiction to highly caffeinated drinks after controlling for potential confounds (e.g., sex, socioeconomic status, regional distribution).

\section{CONCLUSION}

In this study, the high caffeine group was at risk for burnout, ADHD, and impulsiveness; therefore, there is a need for effective evaluation and treatment for ADHD, burnout, and impulsiveness among adults who abuse highly caffeinated drinks.

\section{Acknowledgments}

This manuscript is a revision of the first author's master's thesis from Dankook University 2019.

\section{Conflicts of Interest}

The authors have no potential conflicts of interest to disclose.

\section{Author Contributions}

Conceptualization: Eun Ju Bae, Eun Bi Kim, Bo Ram Choi, Myung Ho Lim. Data curation: Eun Ju Bae, Eun Bi Kim, Bo Ram Choi, Myung Ho Lim. Formal analysis: Eun Ju Bae, Myung Ho Lim. Investigation: Eun Ju Bae, Eun Bi Kim, Bo Ram Choi, Sun Ho Won, Ji Hwan Kim, Sun Min Kim, Hyun Jeong Yoo, Sung Man Bae, Myung Ho Lim. Methodology: Eun Ju Bae, Sung Man Bae, Myung Ho Lim. Project administration: Myung Ho Lim. Supervision: Sung Man Bae, Myung Ho Lim. Validation: Myung Ho Lim. Visualization: Eun Ju Bae. Writing_original draft: Eun Ju Bae. Writing—review \& editing: Myung Ho Lim.

\section{ORCID iDs}

Eun Ju Bae
Eun Bi Kim

Bo Ram Choi

Sun Ho Won

Ji Hwan Kim

Sun Min Kim

Hyun Jeong Yoo

Sung Man Bae

Myung Ho Lim

https://orcid.org/0000-0002-1221-8379

https://orcid.org/0000-0003-4313-3244

https://orcid.org/0000-0003-3680-3641

https://orcid.org/0000-0002-1881-4446

https://orcid.org/0000-0003-3271-9733

https://orcid.org/0000-0002-2777-014X

https://orcid.org/0000-0001-5762-4306

https://orcid.org/0000-0001-5370-4376

\section{REFERENCES}

1) Consumer Safety Center. Survey on safety of energy drink. Korea Consumer Agency;2013 [cited 2019 Feb 15]. Available from URL: http://www.kca.go.kr/brd/m_46/view.do?seq=792\&itm_seq_1=3.

2) Lee JE, Huh W, Choi EJ. Pattern analysis of high-caffeine energy drink consumption and adverse effects among college students in a university. Yakhak Hoeji 2013;57:110-118.

3) National Law Information Center. Article 10 of Enforcement decree of the food sanitation act;2018 [cited 2018 Jan 15]. Available from URL: http://www.law.go.kr/lsInfoP.do?lsiSeq=208599\&ef $\mathrm{Yd}=20191101 \#$.

4) Yoo HS, Shim KH. Survey on the high-caffeine energy drink consumption status of university students in seoul. J East Asian Soc Diet Life 2014;24:407-420.

5) Gunja N, Brown JA. Energy drinks: health risks and toxicity. Med J Aust 2012:196:46-49.

6) Kendler KS, Myers J, Gardner OC. Caffeine intake, toxicity and dependence and lifetime risk for psychiatric and substance use disorders: an epidemiologic and co-twin control analysis. Psychol Med 2006;36:1717-1725.

7) Park WS, Park SU, Kim SA. Correlation between high-caffeine energy drink intake and mental health in high school students. J Korean Soc Sch Health 2016;29:132-139.

8) Stasio MJ, Curry KIM, Wagener AL, Glassman DM. Revving up and staying up: energy drink use associated with anxiety and sleep quality in a college sample. College Stud J 2011;45:738-748.

9) Jang CB, Kim HY. The relationship between attention deficit hyperactivity disorder, dietary habit and caffeine intake in uppergrade elementary school children. Korean J Nutr 2012;45:522-530.

10) Martin CA, Cook C, Woodring JH, Burkhardt G, Guenthner G, Omar HA, et al. Caffeine use: association with nicotine use, aggression, and other psychopathology in psychiatric and pediatric outpatient adolescents. ScientificWorldJournal 2008;8:512-516.

11) Heinz AJ, de Wit H, Lilje TC, Kassel JD. The combined effects of alcohol, caffeine, and expectancies on subjective experience, impulsivity, and risk-taking. Exp Clin Psychopharmacol 2013;21: 222-234.

12) Evren C, Evren B. Energy-drink consumption and its relationship with substance use and sensation seeking among 10th grade students in Istanbul. Asian J Psychiatr 2015;15:44-50.

13) Joudah RA, Gommosani RE, Alsadi DA, Hersi MJ, Abdulmajeid SA. The prevalence of energy drink consumption and its relation to burn out among medical students in Batterjee Medical College in Jeddah, Saudi Arabia. Proceedings of the Researchfora 4th International Conferenc;2017 Apr 2-3;Dubai, UAE. p.17-19.

14) Walsh AL, Gonzalez AM, Ratamess NA, Kang J, Hoffman JR. Improved time to exhaustion following ingestion of the energy drink Amino Impact ${ }^{\mathrm{TM}}$. J Int Soc Sports Nutr 2010;7:14.

15) Seifert SM, Schaechter JL, Hershorin ER, Lipshultz SE. Health effects of energy drinks on children, adolescents, and young adults. Pediatrics 2011;127:511-528.

16) Davis MM, Gance-Cleveland B, Hassink S, Johnson R, Paradis G, Resnicow K. Recommendations for prevention of childhood obesity. Pediatrics 2007;120 Suppl 4:S229-S253. 
17) Miller KE. Energy drinks, race, and problem behaviors among college students. J Adolesc Health 2008;43:490-497.

18) Korean National Information Society Agency. Development of Korean Smartphone Addiction Proneness Scale;2011 [cited 2019 Feb 15]. Available from URL: https://meta.narastat.kr/metasvc/index.do?confmNo=167001\&amp;inputYear=2018.

19) Murphy K, Barkley RA. Preliminary normative data on DSM-IV criteria for adults. ADHD Report 1995;3:6-7.

20) American Psychiatric Association. Diagnostic and statistical manual of mental disorders-IV. 4th ed. Arlington, VA: American Psychiatric Publishing, Inc.;1994.

21) Kim EJ. The validation of Korean adult ADHD scale (K-AADHDS). Korean J Clin Psychol 2003;22:879-911.

22) Korean National Information Society Agency. Development of Korean Smartphone Addiction Proneness Scale for youth and adults. Seoul: Korean National Information Society Agency;2011.

23) Lee YH, Song JY. A study of the reliability and validity of the BDI, SDS, and MMPI-D scales. Korean J Clin Psychol 1991;10: 98-113.

24) Yook SP, Kim ZS. A clinical study on the Korean version of Beck Anxiety Inventory: comparative study of patient and non-patient. Korean J Clin Psychol 1997;16:185-197.

25) Kang JH, Kim CW. Evaluating Applicability of Maslach Exhaus- tion Inventory among university hospitals nurses. Korean J Adult Nurs 2012;24:31-37.

26) Lee YH. Relations between attributional style, life events, event attribution, hopelessness and depression. Seoul: Seoul National Univ;1993.

27) Lee HS. Impulsivity test. Seoul: Korean Guidance;1992.

28) Lee SH, Kang MK. The investigation of high-caffeineated beverages consumption among college students. AJMAHS 2015;5:1-10.

29) Walker LR, Abraham AA, Tercyak KP. Adolescent caffeine use, ADHD, and cigarette smoking. J Child Health Care 2010;39:73-90.

30) Ahola K, Honkonen T, Kivimäki M, Virtanen M, Isometsä E, Aromaa A, et al. Contribution of burnout to the association between job strain and depression: the health 2000 study. J Occup Environ Med 2006;48:1023-1030.

31) Komaroff AL, Fagioli LR, Doolittle TH, Gandek B, Gleit MA, Guerriero RT, et al. Health status in patients with chronic fatigue syndrome and in general population and disease comparison groups. Am J Med 1996;101:281-290.

32) Woolston JL, Rosenthal SL, Riddle MA, Sparrow SS, Cicchetti D, Zimmerman LD. Childhood comorbidity of anxiety/affective disorders and behavior disorders. J Am Acad Child Adolesc Psychiatry 1989;28:707-713. 